\title{
Prevalence and associated factors of psychological distress among a national sample of in-school adolescents in Morocco
}

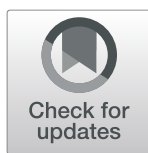

Supa Pengpid ${ }^{1,2}$ and Karl Peltzer ${ }^{3^{*}}$ (1)

\begin{abstract}
Background: The goal of the study was to estimate the prevalence and correlates of psychological distress among adolescent school children in Morocco.

Methods: Nationally representative cross-sectional data were analysed from 6745 adolescents (15 years median age) that responded to questions on a two-item measure of psychological distress from "2016 Morocco Global School-Based Student Health Survey (GSHS)."

Results: The prevalence of psychological distress was 23.3, 18.0\% among males and 29.2\% among females. In adjusted logistic regression analysis, female sex, older age, bullying victimization, infrequently physically attacked, frequent participation in physical fights, having no close friends, frequent experience of hunger, parental emotional neglect, parental disrespect of privacy, school truancy, sedentary behaviour and having sustained a single or multiple serious injuries (past year) were associated with psychological distress. In addition, in unadjusted analysis, low peer support, parents never check homework, exposure to passive smoking, substance use (current tobacco use, current cannabis use and ever used amphetamine), frequent soft drink and frequent fast food consumption were positively and fruit and vegetable intake was negatively associated with psychological distress.
\end{abstract}

Conclusion: Almost one in four students reported psychological distress and several associated factors were identified which can aid prevention and control strategies.

Keywords: Risk behaviour, Social factors, Psychological distress, Adolescents, Morocco

\section{Background}

"Mental health conditions, including depression and anxiety, account for $16 \%$ of the global burden of disease and injury in people aged 10-19 years." [1]. In children and adolescents, the "worldwide-pooled prevalence of mental disorders was $13.4 \%$, including any anxiety disorder $6.5 \%$ and any depressive disorder 2.6\%" [2]. "First onset of mental disorders usually occurs in childhood or adolescence" [3]. "Adolescence is a critical period

\footnotetext{
*Correspondence: kfpeltzer@gmail.com

${ }^{3}$ Department of Psychology, University of the Free State, Bloemfontein, South Africa

Full list of author information is available at the end of the article
}

characterised by vulnerability to psychological distress, and is therefore an important time for promotion of psychological well-being and early mental health intervention, in order to safeguard against the development of mental health issues" [4]. According to the American Psychological Association (APA) [5], psychological distress is "a set of painful mental and physical symptoms that are associated with normal fluctuations of mood in most people. It is thought to be what is assessed by many putative self-report measures of depression and anxiety." For example, the Kessler Psychological Distress Scale includes "symptoms of depression, anxiety, stress, and somatic complaints" [6]. Psychological distress has

(C) The Author(s). 2020 Open Access This article is licensed under a Creative Commons Attribution 4.0 International License, which permits use, sharing, adaptation, distribution and reproduction in any medium or format, as long as you give appropriate credit to the original author(s) and the source, provide a link to the Creative Commons licence, and indicate if changes were made. The images or other third party material in this article are included in the article's Creative Commons licence, unless indicated otherwise in a credit line to the material. If material is not included in the article's Creative Commons licence and your intended use is not permitted by statutory regulation or exceeds the permitted use, you will need to obtain permission directly from the copyright holder. To view a copy of this licence, visit http://creativecommons.org/licenses/by/4.0/ The Creative Commons Public Domain Dedication waiver (http://creativecommons.org/publicdomain/zero/1.0/) applies to the data made available in this article, unless otherwise stated in a credit line to the data. 
been assessed with different scales, such as the "Psychological Distress Scale", K-6 or K-10 [6, 7], the "General Health Questionnaire (GHQ-12)" [8] and several different psychological distress items, such as anxiety, loneliness, sadness and suicide plan [9], or no close friends, anxiety, loneliness, suicidal ideation and attempt [10].

The prevalence of psychological distress among adolescents in Eastern Mediterranean countries was 27.7\% ( $\geq 2$ items of no close friends, loneliness, anxiety, suicidal ideation, and suicide attempt) in Afghanistan [11], 16.8\% ( $\geq 14$ scores on GHQ-28) in Egypt [12], 17.7\% ( $\geq 3$ items of worthless, anxious, angriness, confusion, and insomnia) in Iran [13], and $17.2 \%$ probable depression and $21.4 \%$ probable anxiety ( $\geq 10$ scores and $\geq 12$ scores on the Hospital Anxiety and Depression Scale, respectively) in Pakistan [14]. The proportion of moderate to severe psychological distress (measured with the Kessler $\mathrm{K}-10$ ) was $23.0 \%$ in a community-based study among adolescents $(N=1000)$ in Tanzania [15] and 10.5\% among school-going adolescents in India [16], and the prevalence of psychological distress measured with the GHQ was $35.1 \%$ among adolescents in Ontario, Canada [17], and $24.2 \%$ among urban out of school adolescents $(N=480)$ in Nigeria [18]. In Zambia, the prevalence of psychological distress among adolescents was $15.7 \%$ (scoring 3 of 4 items, anxiety, loneliness, sadness, and suicide plan [9], in Tanzania among adolescents $20.6 \%$ had single psychological distress and $10.3 \%$ had multiple psychological distress (based on five items: anxiety, loneliness, no close friends, suicidal ideation and suicide attempt) [10], and in four Asian countries psychological distress was $32.9 \%$ (presence of any item: suicidal ideation, plan and suicide attempt, loneliness, sadness and anxiety) [19]. In a local survey among secondary school students in an urban area in Morocco (Tetouan), the most common mental problems "were memory problems, concentration difficulties, restlessness, fear, nervosity and feelings of inadequacy during interpersonal interactions." [20]. There is a lack of national data on the prevalence and correlates of psychological distress among adolescents in Morocco. In order to prevent and control psychological distress in adolescent populations, it is vital to assess its prevalence and risk factors [21].

Factors associated with psychological distress among adolescents can be divided into social distresses, socioenvironmental factors and health risk behaviours [22], and may include, as reviewed in Pengpid and Peltzer [10], sociodemographic factors (older age, female sex), social distresses (interpersonal violence), socioenvironmental factors (experience of hunger, low peer and low parental support and school truancy) and health risk behaviours (substance use, sedentary behaviour, sexual behaviour and injury). This investigation aimed at estimating the prevalence and correlates of psychological distress among adolescents in Morocco.

\section{Methods}

\section{Sources of data}

Nationally representative cross-sectional data from the "2016 Morocco GSHS" were analyzed [23]. More detailed information on the survey methodology and the data can be accessed [23]; the overall response rate was 91\% [23]. The sampling approach included a two stage sampling design, including schools and classes. All school students present in a selected class room were eligible to participate by filling in a self-administered anonymous questionnaire [23].

\section{Measures}

The questionnaire (the questions, response options and coding) used is shown in supplementary file 1 [23].

\section{Outcome variable}

Psychological distress was assessed with two items, 1) "During the past 12 months, how often have you been so worried about something that you could not sleep at night?" and 2) "During the past 12 months, how often have you felt lonely?" Response options included and were coded as "Never $=0$, Rarely $=1$, Sometimes $=1$, Most of the time $=2$, Always $=3$ " Scores of the two items were summed and scores three or more were defined as psychological distress, following the scoring of other 2-item mental health screeners, the "Patient Health Questionnaire-2 (PHQ-2)" [24], and the "Generalized Anxiety Disorder-2 (GAD-2)" [25, 26]. Cronbach alpha for the 2-item "psychological distress" measure was 0.66 in this sample. The inter-item correlation was 0.44 , and the item-total correlations ranged from .82 to .90 .

\section{Co-variates}

Sociodemographic variables included sex and age group.

Psychosocial distress items included the number of close friends, the number of days having been bullied in the past 30 days, the number of times having been physically attacked in the past 12 months, and the number of times having been involved in a physical fight in the past 12 months.

Social-environmental variables included, the frequency of feeling hungry in the past 30 days, the extend of peer support, parental disrespect of privacy, number of days exposed secondary smoke in the past 7 days, number of days truancy in the past 30 days, and parental emotional neglect was defined as never "parental or guardian understanding of your problems and worries? AND never parents or guardians really know what you were doing with your free time when you were not at school or work?" [27].

Health risk behaviour items included, current tobacco use, current cannabis use, ever used amphetamine, number of hours spent sedentary during leisure time, 
number of times seriously injured in the past 12 months, number of soft drinks consumed in a day, number of days having had fast food in the past week, and fruit and vegetable intake per day.

\section{Data analysis}

Statistical analyses were done with "STATA software version 15.0 (Stata Corporation, College Station, Texas, USA)," taking into account the complex study design. The data were described using frequencies, means, and standard deviations. Pearson Chi-square tests were used for testing differences in proportions. In addition, Cronbach alpha coefficients were calculated and Principal Component Analysis with the 2-item psychological distress measure. Unadjusted and adjusted (with all covariates) logistic regression analyses were used to assess predictors of psychological distress. Co-variates included age group, social distress (no close friends, being bullied, physically attacked, and participation in physical fight), social-environmental factors (experience of hunger, low peer support, lack of parental support, passive smoking, and school truancy), and health risk behaviours (tobacco use, cannabis use, amphetamine use, sedentary behaviour, injury, soft drink intake, fast food consumption, and fruit and vegetable intake). They were selected based on literature review [10]. Missing values $(<3.7 \%$ on any variable) were not included in the analysis.

\section{Results}

\section{Factor analysis of the 2-item psychological distress measure}

The Kaiser-Meyer-Olkin was .500, showing the adequacy of the sample, and the Bartlett's Test of Sphericity (549, 195.047, $\mathrm{df}=1, p<0.001$ ) found that the factor analysis was justified. One component with eigenvalues larger than one (1.44) was extracted and named "psychological distress" explaining $72.21 \%$ of the variance (see Table 1).

\section{Sample and psychological distress characteristics}

The sample comprised 6745 school adolescents (15 years median age, 3 years interquartile range), $46.2 \%$ were female and $9.2 \%$ were mostly or always hungry. One in ten of the participants (10.2\%) had no close friends, $19.8 \%$ were lonely, $18.2 \%$ had frequently been in a physical fight, $11.7 \%$ had frequently been attacked, and $14.7 \%$ had frequently been bullied. More than one in ten students (13.5\%) reported current tobacco use, 6.3\% current cannabis use, $7.8 \%$ had ever used amphetamine, 9.1\% were daily exposed to secondary smoke, $17.2 \%$ had daily two or more soft drinks, $12.1 \%$ had multiple injuries (past year), 32.5\% engaged in sedentary behaviour, 26.9\% had on three or more days fast food in the past week and $33.0 \%$ had five or more servings of fruit and vegetables per day. One in ten of the students $(10.1 \%)$ reported
Table 1 Factor loadings of the 2-item psychological distress measure among adolescents in Morocco

\begin{tabular}{ll}
\hline Psychological distress: 2 items & Component \\
& 1 \\
& \\
$\begin{array}{l}\text { 1. During the past } 12 \text { months, how often have you been } \\
\text { so worried about something that you could not sleep at } \\
\text { night? }\end{array}$ & .85 \\
$\begin{array}{l}\text { 2. During the past } 12 \text { months, how often have you felt } \\
\text { lonely? }\end{array}$ & .85 \\
Eigenvalue & 1.44 \\
Percent of variance & 72.21 \\
Factor (items: 1 and 2): Psychological distress & \\
\hline
\end{tabular}

frequently truancy, $45.4 \%$ had low peer support, $24.1 \%$ experienced parental emotional neglect, $29.5 \%$ had parents who never checked on their home work, and 13.1\% had parents who mostly or always disrespected their privacy. Almost one in four students (23.3\%) reported psychological distress, 29.2\% among females and 18.0\% among males (see Table 2).

\section{Associations with psychological distress among both sexes}

Table 3 shows the unadjusted and adjusted associations between sociodemographic factors, social distress items, social-environmental factors and health risk behaviours with psychological distress. Compared to students aged 13 years or younger, older adolescents aged 16 years and older reported $129 \%(\mathrm{AOR}=2.29 ; 95 \% \mathrm{CI}=1.73-3.03)$ higher prevalence of psychological distress. Male adolescents were $70 \%(\mathrm{AOR}=0.30 ; 95 \% \mathrm{CI}=0.23-0.40)$ less likely having psychological distress than female adolescents do. Social distress factors associated with psychological distress were frequent bullying victimization $(\mathrm{AOR}=2.92 ; 95 \% \mathrm{CI}=2.16-3.94)$, having no close friends $(\mathrm{AOR}=1.98 ; 95 \% \mathrm{CI}=1.44-2.73)$, frequent involvement on physical fighting $(\mathrm{AOR}=1.89 ; 95 \% \mathrm{CI}=$ 1.34-2.67), and having been attacked once $(\mathrm{AOR}=1.47$; $95 \% \mathrm{CI}=1.08-2.00)$. Social-environmental factors associated with psychological distress were frequent truancy $(\mathrm{AOR}=1.87 ; 95 \% \mathrm{CI}=1.23-2.83)$, frequent experience of hunger $(\mathrm{AOR}=1.49 ; 95 \% \mathrm{CI}=1.07-2.08)$, parental emotional neglect $(\mathrm{AOR}=1.37 ; 95 \% \mathrm{CI}=1.07-1.75)$, and parental disrespect of privacy $(\mathrm{AOR}=1.33 ; 95 \% \mathrm{CI}=$ 1.01-1.77). Regarding health risk behaviours, compared to students who had not sustained a serious injury in the past year, students who had multiple injuries were $122 \%$ $(\mathrm{AOR}=2.22 ; 95 \% \mathrm{CI}=1.69-2.93)$ more likely to have psychological distress. Compared to students who were less than $3 \mathrm{~h}$ a day engaged in leisure-time sedentary behaviour, students who engaged eight or more hours a day in leisure-time sedentary behaviour had $71 \%(\mathrm{AOR}=1.71$; $95 \% \mathrm{CI}=1.24-2.36)$ more likely psychological distress. In 
Table 2 Sample and psychological distress characteristics among adolescents in Morocco

\begin{tabular}{|c|c|c|c|c|}
\hline \multirow[t]{3}{*}{ Variable } & \multirow{3}{*}{$\begin{array}{l}\text { Sample } \\
\text { N (\%) }\end{array}$} & \multicolumn{3}{|c|}{ Psychological distress } \\
\hline & & Both sexes & Male & Female \\
\hline & & $\%$ & $\%$ & $\%$ \\
\hline \multicolumn{5}{|l|}{ Socio-demographics } \\
\hline All & 6745 & 23.3 & 18.0 & 29.2 \\
\hline \multicolumn{5}{|l|}{ Age in years } \\
\hline 13 or less & $1863(26.8)$ & 16.4 & 16.5 & 14.9 \\
\hline $14-15$ & $2212(31.9)$ & 21.9 & 14.4 & 28.6 \\
\hline 16 or more & $2558(41.3)$ & 30.3 & 21.2 & 41.0 \\
\hline \multicolumn{5}{|l|}{ Psychosocial distress } \\
\hline No close friends & $698(10.2)$ & 37.6 & 32.3 & 48.3 \\
\hline \multicolumn{5}{|l|}{ Bullied in past month } \\
\hline 0 days & $3869(61.5)$ & 15.9 & 11.1 & 19.7 \\
\hline 1 or 2 days & $1511(23.8)$ & 29.3 & 20.0 & 40.5 \\
\hline $3-30$ days & $955(14.7)$ & 45.5 & 36.7 & 59.6 \\
\hline \multicolumn{5}{|l|}{ Physically attacked in past year } \\
\hline 0 times & $5080(76.7)$ & 20.2 & 14.1 & 25.6 \\
\hline time & $795(11.5)$ & 32.5 & 26.6 & 40.1 \\
\hline 2 or more times & $793(11.7)$ & 38.8 & 31.6 & 53.1 \\
\hline \multicolumn{5}{|l|}{ In physical fight in past year } \\
\hline 0 times & $64.2(63.4)$ & 21.7 & 14.1 & 26.7 \\
\hline 1 time & $18.4(18.4)$ & 23.2 & 15.1 & 35.2 \\
\hline 2 or more times & $17.4(18.2)$ & 31.8 & 27.4 & 46.5 \\
\hline \multicolumn{5}{|l|}{ Social-environmental factors } \\
\hline Mostly/always feeling hungry & $680(9.2)$ & 38.5 & 36.6 & 42.7 \\
\hline Low peer support & $3085(45.4)$ & 25.6 & 18.9 & 34.0 \\
\hline Parental emotional neglect & $1655(24.1)$ & 25.1 & 20.2 & 34.2 \\
\hline Parents never check home work & $2029(29.5)$ & 25.7 & 20.2 & 33.5 \\
\hline Parents disrespect privacy & $818(13.1)$ & 30.7 & 18.0 & 36.0 \\
\hline \multicolumn{5}{|l|}{ Passive smoking in past week } \\
\hline 0 days & $3908(59.0)$ & 20.8 & 14.8 & 26.2 \\
\hline $1-6$ days & $2104(31.9)$ & 24.7 & 18.4 & 32.2 \\
\hline All 7 days & $580(9.1)$ & 38.2 & 30.7 & 43.4 \\
\hline \multicolumn{5}{|l|}{ School truancy (past month) } \\
\hline 0 days & $4584(69.3)$ & 20.6 & 14.5 & 25.7 \\
\hline $1-2$ days & $1317(20.6)$ & 28.4 & 20.0 & 37.5 \\
\hline 3 or more days & $644(10.1)$ & 36.1 & 31.6 & 48.7 \\
\hline \multicolumn{5}{|l|}{ Health risk behaviours } \\
\hline Current tobacco use & $913(13.5)$ & 31.9 & 29.8 & 40.4 \\
\hline Current cannabis use & $453(6.3)$ & 34.1 & 37.1 & 37.6 \\
\hline Ever used amphetamine & $464(7.8)$ & 37.2 & 36.6 & 47.9 \\
\hline \multicolumn{5}{|c|}{ Leisure time sedentary behaviour/day } \\
\hline$<3 \mathrm{~h}$ & $4574(67.5)$ & 21.2 & 16.3 & 24.9 \\
\hline $3-4 h$ & $1100(18.7)$ & 26.2 & 15.6 & 36.3 \\
\hline $5-7 h$ & $485(8.4)$ & 31.6 & 24.6 & 41.4 \\
\hline
\end{tabular}


Table 2 Sample and psychological distress characteristics among adolescents in Morocco (Continued)

\begin{tabular}{|c|c|c|c|c|}
\hline \multirow[t]{3}{*}{ Variable } & \multirow{3}{*}{$\begin{array}{l}\text { Sample } \\
\text { N (\%) }\end{array}$} & \multicolumn{3}{|c|}{ Psychological distress } \\
\hline & & Both sexes & Male & Female \\
\hline & & $\%$ & $\%$ & $\%$ \\
\hline$\geq 8 \mathrm{~h}$ & $306(5.4)$ & 39.8 & 36.9 & 43.7 \\
\hline \multicolumn{5}{|c|}{ Injury in past 12 months } \\
\hline 0 times & $4251(68.4)$ & 18.1 & 12.1 & 22.2 \\
\hline 1 time & $1206(19.5)$ & 29.7 & 23.2 & 39.0 \\
\hline 2 or more times & $735(12.1)$ & 39.5 & 32.2 & 58.6 \\
\hline \multicolumn{5}{|l|}{ Soft drink intake/day } \\
\hline 0 & $4488(67.4)$ & 23.5 & 16.6 & 29.4 \\
\hline 1 & $1033(15.3)$ & 22.0 & 17.7 & 25.8 \\
\hline 2 & $423(6.6)$ & 22.9 & 21.2 & 31.2 \\
\hline 3 or more & $706(10.6)$ & 27.1 & 23.5 & 31.3 \\
\hline \multicolumn{5}{|c|}{ Fast food consumption/week } \\
\hline 0 days & $2486(35.5)$ & 22.3 & 15.9 & 26.7 \\
\hline 1 & $1579(23.7)$ & 22.4 & 16.7 & 26.8 \\
\hline 2 & $899(13.8)$ & 23.5 & 18.3 & 28.8 \\
\hline 3-7 days & $1718(26.9)$ & 27.0 & 21.5 & 35.6 \\
\hline \multicolumn{5}{|c|}{ Fruit and vegetable intake } \\
\hline$<1$ serving/day & $560(8.2)$ & 32.3 & 24.2 & 43.0 \\
\hline 1 or 2 & $2222(34.1)$ & 25.0 & 18.8 & 31.3 \\
\hline 3 or 4 & $1591(24.7)$ & 20.5 & 14.1 & 26.7 \\
\hline 5 or more & $2158(33.0)$ & 22.0 & 17.9 & 25.5 \\
\hline
\end{tabular}

addition, in unadjusted analyses, low peer support, parents never check home work, exposure to passive smoking, substance use (current tobacco use, current cannabis use and ever used amphetamine), frequent soft drink and frequent fast food consumption were positively and fruit and vegetable intake was negatively associated with psychological distress (see Table 3).

\section{Associations with psychological distress among boys and girls}

Compared to 13 or less year-old girls, 16 or more year-old girls were 3.5 times more likely to have psychological distress. Among both boys and girls, frequent bullying victimization, frequently having been in a physical fight, having no close friends, frequent truancy and frequent injury increased the odds for psychological distress. Boys who frequently experienced hunger were $114 \%(\mathrm{AOR}=2.14 ; 95 \% \mathrm{CI}=1.31-$ 3.48) more likely to have psychological distress, and girls who had low peer support were $23 \%$ (AOR = 1.23; $95 \% \mathrm{CI}=1.05-1.44$ ) and engaged 8 or more hours in leisure-time sedentary behaviour were $91 \%$ $(\mathrm{AOR}=1.91 ; 95 \% \mathrm{CI}=1.14-3.20)$ more likely to have psychological distress (see Table 4).

\section{Discussion}

The current study aimed at estimating the prevalence and correlates of psychological distress in school adolescents in Morocco. The prevalence of past 12-month psychological distress $(23.3 \%)$ in this study, which is similar to some previous studies among adolescents, e.g., in Tanzania (23.0\%) [15], Nigeria (24.2\%) [18], lower than in Afghanistan (27.7\%) [11], in United Arab Emirates (28\% anxiety disorders) [28], in Lebanon (Beirut) $(26.1 \%$ mental disorders [29], in Canada (35.1\%) [17], and higher than in Egypt (16.8\%) [12], India (10.5\%) [16], and Iran (17.7\%) [13]. Other previous studies among adolescents in the Morocco seem to confirm that psychological distress is common [20], calling for strategies and programmes to prevent and control psychological distress in this adolescent population in Morocco.

The study showed that being female increased the odds for psychological distress, which was also found in some previous investigations [11]. Generally, "girls are more likely than boys to report internalising problems such as psychological distress, depression, and anxiety." $[4,30,31]$. The study showed that older age, in particular among girls, increased the odds for psychological distress. Similar results were found in a study among adolescents in India [16]. Possible reasons for higher 
Table 3 Associations with psychological distress in both sexes

\begin{tabular}{|c|c|c|c|c|}
\hline Variable & Unadjusted Odds Ratio $(95 \% \mathrm{Cl})$ & $P$-value & Adjusted Odds Ratio $(95 \% \mathrm{Cl})$ & $P$-value \\
\hline \multicolumn{5}{|l|}{ Socio-demographics } \\
\hline \multicolumn{5}{|l|}{ Age in years } \\
\hline 13 or less & 1 (Reference) & & 1 (Reference) & \\
\hline $14-15$ & $1.46(1.19,1.79)$ & $<0.001$ & $1.42(1.07,1.89)$ & 0.016 \\
\hline 16 or more & $2.26(1.89,2.71)$ & $<0.001$ & $2.29(1.73,3.03)$ & $<0.001$ \\
\hline \multicolumn{5}{|l|}{ Gender } \\
\hline Female & 1 (Reference) & & 1 (Reference) & \\
\hline Male & $0.53(0.45,0.63)$ & $<0.001$ & $0.30(0.23,0.40)$ & $<0.001$ \\
\hline \multicolumn{5}{|l|}{ Social distress } \\
\hline No close friends & $2.53(0.25,3.24)$ & $<0.001$ & $1.98(1.44,2.73)$ & $<0.001$ \\
\hline \multicolumn{5}{|l|}{ Bullied in past month } \\
\hline 0 days & 1 (Reference) & & 1 (Reference) & \\
\hline 1 or 2 days & $2.22(1.87,2.62)$ & $<0.001$ & $1.95(1.58,2.40)$ & $<0.001$ \\
\hline $3-30$ days & $4.70(3.70,5.98)$ & $<0.001$ & $2.92(2.16,3.94)$ & $<0.001$ \\
\hline \multicolumn{5}{|l|}{ Physically attacked in past year } \\
\hline 0 times & 1 (Reference) & & 1 (Reference) & \\
\hline 1 time & $1.89(1.51,2.37)$ & $<0.001$ & $1.47(1.08,2.00)$ & 0.015 \\
\hline 2 or more times & $2.63(2.13,3.23)$ & $<0.001$ & $1.02(0.72,1.46)$ & 0.987 \\
\hline \multicolumn{5}{|l|}{ In physical fight in past year } \\
\hline 0 times & 1 (Reference) & & 1 (Reference) & \\
\hline 1 time & $1.00(0.83,1.21)$ & 0.989 & $1.20(0.89,1.60)$ & 0.232 \\
\hline 2 or more times & $1.66(1.34,2.04)$ & $<0.001$ & $1.89(1.34,2.67)$ & $<0.001$ \\
\hline \multicolumn{5}{|l|}{ Social-environmental factors } \\
\hline Mostly/always feeling hungry & $2.30(1.78,2.98)$ & $<0.001$ & $1.49(1.07,2.08)$ & 0.019 \\
\hline Low peer support & $1.27(1.11,1.45)$ & $<0.001$ & $1.15(0.97,1.35)$ & 0.098 \\
\hline Parental emotional neglect & $1.29(1.09,1.52)$ & 0.003 & $1.37(1.07,1.75)$ & 0.014 \\
\hline Parents never check home work & $1.27(1.02,1.58)$ & 0.031 & $1.11(0.90,1.36)$ & 0.326 \\
\hline Parents disrespect privacy & $1.27(1.07,1.50)$ & 0.006 & $1.33(1.01,1.77)$ & 0.045 \\
\hline \multicolumn{5}{|l|}{ Passive smoking in past week } \\
\hline 0 days & 1 (Reference) & & 1 (Reference) & \\
\hline $1-6$ days & $1.18(1.04,1.33)$ & 0.010 & $0.89(0.67,1.17)$ & 0.388 \\
\hline All 7 days & $2.17(1.78,2.64)$ & $<0.001$ & $1.19(0.82,1.71)$ & 0.362 \\
\hline \multicolumn{5}{|l|}{ School truancy (past month) } \\
\hline 0 days & 1 (Reference) & & 1 (Reference) & \\
\hline $1-2$ days & $1.49(1.28,1.74)$ & $<0.001$ & $1.37(1.08,1.74)$ & 0.009 \\
\hline 3 or more days & $2.35(1.81,3.04)$ & $<0.001$ & $1.87(1.23,2.83)$ & 0.004 \\
\hline \multicolumn{5}{|l|}{ Health risk behaviours } \\
\hline Current tobacco use & $1.67(1.34,2.07)$ & $<0.001$ & $0.68(0.48,1.01)$ & 0.098 \\
\hline Current cannabis use & $2.07(1.56,2.74)$ & $<0.001$ & $0.96(0.57,1.61)$ & 0.877 \\
\hline Ever used amphetamine & $2.34(1.86,2.94)$ & $<0.001$ & $1.58(0.91,2.75)$ & 0.100 \\
\hline \multicolumn{5}{|c|}{ Leisure time sedentary behaviour/day } \\
\hline$<3 \mathrm{~h}$ & 1 (Reference) & & 1 (Reference) & \\
\hline $3-4 h$ & $1.29(1.09,1.53)$ & 0.003 & $1.04(0.73,1.48)$ & 0.820 \\
\hline $5-7 \mathrm{~h}$ & $1.74(1.42,2.14)$ & $<0.001$ & $1.68(1.23,2.30)$ & $<0.001$ \\
\hline
\end{tabular}


Table 3 Associations with psychological distress in both sexes (Continued)

\begin{tabular}{|c|c|c|c|c|}
\hline Variable & Unadjusted Odds Ratio $(95 \% \mathrm{Cl})$ & $P$-value & Adjusted Odds Ratio (95\% Cl) & $P$-value \\
\hline$\geq 8 \mathrm{~h}$ & $2.70(2.10,3.46)$ & $<0.001$ & $1.71(1.24,2.36)$ & $<0.001$ \\
\hline \multicolumn{5}{|c|}{ Injury in past 12 months } \\
\hline 0 times & 1 (Reference) & & 1 (Reference) & \\
\hline 1 time & $2.00(1.74,2.29)$ & $<0.001$ & $1.50(1.20,1.87)$ & $<0.001$ \\
\hline 2 or more times & $3.23(2.73,3.82)$ & $<0.001$ & $2.22(1.69,2.93)$ & $<0.001$ \\
\hline \multicolumn{5}{|l|}{ Soft drink intake/day } \\
\hline 0 & 1 (Reference) & & 1 (Reference) & \\
\hline 1 & $0.95(0.74,1.22)$ & 0692 & $0.85(0.61,1.19)$ & 0.335 \\
\hline 2 & $1.21(0.91,1.63)$ & 0.194 & $0.94(0.65,1.36)$ & 0.756 \\
\hline 3 or more & $1.28(1.06,1.54)$ & 0.011 & $1.00(0.60,1.64)$ & 0.986 \\
\hline \multicolumn{5}{|c|}{ Fast food consumption/week } \\
\hline 0 days & 1 (Reference) & & 1 (Reference) & \\
\hline 1 & $0.96(0.80,1.14)$ & 0.618 & $0.92(0.67,1.26)$ & 0.600 \\
\hline 2 & $1.04(0.90,1.20)$ & 0.593 & $0.86,0.65,1.13)$ & 0.282 \\
\hline $3-7$ days & $1.52(1.19,1.94)$ & $<0.001$ & $1.04(0.75,1.44)$ & 0.828 \\
\hline \multicolumn{5}{|c|}{ Fruit and vegetable intake } \\
\hline$<1$ serving/day & 1 (Reference) & & 1 (Reference) & \\
\hline 1 or 2 & $0.66(0.51,0.86)$ & 0.003 & $0.99(0.74,1.33)$ & 0.965 \\
\hline 3 or 4 & $0.51(0.39,0.67)$ & $<0.001$ & $0.86(0.61,1.20)$ & 0.374 \\
\hline 5 or more & $0.55(0.42,0.72)$ & $<0.001$ & $0.88(0.61,1.28)$ & 0.499 \\
\hline
\end{tabular}

Cl Confidence Interval

psychological distress among older than younger adolescents include increasing demands, physical and psychosocial changes $[4,32,33]$. In addition, older adolescents in this study were more likely to be injured $(p=0.009)$, engage in substance use (tobacco use: $p<0.001$, cannabis use: $p=0.008$, amphetamine use: $p=0.003)$, truancy $(p<0.001)$, sedentary behaviour $(p<0.001)$, passive smoking $(p<0.001)$, had no close friends $(p<0.001)$, frequent fast food intake $(p=0.003)$ and ate less fruit and vegetables $(p<0.001)$ than younger adolescents, which may have contributed to increased psychological distress among older adolescents.

In line with former research findings [9, 10, 19, 34], this survey showed that having social distress, such as having no close friends, bullying victimization, infrequently physically attacked and frequently involved in physical fighting increased the odds for psychological distress. Students exposed to interpersonal violence victimization may worry about further or future victimization increasing psychological distress. In addition, our study findings demonstrate that students who had been frequently bullied had the highest odds for psychological distress, which concurs with former research [35]. This finding may highlight the relevance of anti-bullying programme activities in order to ameliorate psychological distress. "The design and implementation of school-based anti-bullying programmes in the Arab world to reduce the harmful effects of bullying are lacking." [36] However, Morocco has "implemented coordinated national action plans to address violence against children, enforced legislation to protect victims, and promoted programmes aimed at changing societal beliefs and attitudes around violence." [37]

Several social-environmental factors (experience of hunger, in particular among boys, parental emotional neglect, parental disrespect of privacy, infrequent truancy and in unadjusted analysis passive smoking, low peer support, in particular among girls, and parents never check homework) were found associated with psychological distress. These results are consistent with various previous investigations $[9,10,19,38,39]$ and call for programmes improving parental and peer support and food security. A previous review provides evidence that "parental training and school-based interventions can reduce symptoms of common mental disorders in adolescents" [40].

In terms of health risk behaviours, high sedentary behaviour (in particular among girls) and having experienced single and multiple serious injuries, and in unadjusted analysis frequent fast food consumption, infrequent fruit and vegetable intake, frequent soft drink, having no close friends, and substance use increased the odds for having psychological distress. These findings concur with previous studies [10, 17, 4145]. Since this study did not assess the type of 
Table 4 Associations with psychological distress among males and females

\begin{tabular}{|c|c|c|c|c|}
\hline \multirow[t]{2}{*}{ Variable } & \multicolumn{2}{|l|}{ Male } & \multicolumn{2}{|l|}{ Female } \\
\hline & Adjusted Odds Ratio $(95 \% \mathrm{Cl})$ & $p$-value & Adjusted Odds Ratio $(95 \% \mathrm{Cl})$ & $p$-value \\
\hline \multicolumn{5}{|l|}{ Socio-demographics } \\
\hline \multicolumn{5}{|l|}{ Age in years } \\
\hline 13 or less & 1 (Reference) & & 1 (Reference) & \\
\hline $14-15$ & $0.77(0.46,1.29)$ & 0.321 & $2.12(1.48,3.03)$ & $<0.001$ \\
\hline 16 or more & $1.23(0.75,2.02)$ & 0.414 & $3.52(2.50,4.95)$ & $<0.001$ \\
\hline \multicolumn{5}{|l|}{ Social distress } \\
\hline No close friends & $2.88(1.73,4.81)$ & $<0.001$ & $1.61(1.14,2.28)$ & 0.007 \\
\hline \multicolumn{5}{|l|}{ Bullied in past month } \\
\hline 0 days & 1 (Reference) & & 1 (Reference) & \\
\hline 1 or 2 days & $1.61(1.08,2.39)$ & 0.020 & $2.24(1.60,3.15)$ & $<0.001$ \\
\hline $3-30$ days & $2.50(1.63,3.82)$ & $<0.001$ & $3.41(2.24,5.18)$ & $<0.001$ \\
\hline \multicolumn{5}{|l|}{ Physically attacked in past year } \\
\hline 0 times & 1 (Reference) & & 1 (Reference) & \\
\hline 1 time & $1.64(1.00,2.70)$ & 0.051 & $1.17(0.73,1.88)$ & 0.516 \\
\hline 2 or more times & $1.02(0.67,1.57)$ & 0.921 & $1.19(0.62,2.31)$ & 0.594 \\
\hline \multicolumn{5}{|l|}{ In physical fight in past year } \\
\hline 0 times & 1 (Reference) & & 1 (Reference) & \\
\hline 1 time & $1.12(0.77,1.64)$ & 0.549 & $1.38(0.94,2.03)$ & 0.099 \\
\hline 2 or more times & $1.98(1.33,2.96)$ & $<0.001$ & $1.86(1.21,2.87)$ & 0.005 \\
\hline \multicolumn{5}{|l|}{ Social-environmental factors } \\
\hline Mostly/always feeling hungry & $2.14(1.31,3.48)$ & 0.002 & $1.05(0.69,1.61)$ & 0.805 \\
\hline Low peer support & $0.99(0.77,1.27)$ & 0.915 & $1.23(1.05,1.44)$ & 0.013 \\
\hline Parental emotional neglect & $1.28(0.91,1.81)$ & 0.404 & $1.43(0.98,2.08)$ & 0.063 \\
\hline Parents never check home work & $1.36(0.99,1.86)$ & 0.057 & $0.92(0.68,1.26)$ & 0.610 \\
\hline Parents disrespect privacy & $1.20(0.78,1.85)$ & 0.404 & $1.32(0.88,2.00)$ & 0.182 \\
\hline \multicolumn{5}{|l|}{ Passive smoking in past week } \\
\hline 0 days & 1 (Reference) & & 1 (Reference) & \\
\hline $1-6$ days & $0.88(0.67,1.14)$ & 0.329 & $0.90(0.65,1.25)$ & 0.519 \\
\hline All 7 days & $1.15(0.77,1.71)$ & 0.494 & $1.27(0.76,2.12)$ & 0.353 \\
\hline \multicolumn{5}{|l|}{ School truancy (past month) } \\
\hline 0 days & 1 (Reference) & & 1 (Reference) & \\
\hline $1-2$ days & $1.58(1.15,2.17)$ & 0.006 & $1.28(0.92,1.79)$ & 0.146 \\
\hline 3 or more days & $2.12(1.28,3.51)$ & 0.004 & $2.01(1.04,3.89)$ & 0.039 \\
\hline \multicolumn{5}{|l|}{ Health risk behaviours } \\
\hline Current tobacco use & $0.81(0.53,1.24)$ & 0.326 & $0.50(0.19,1.28)$ & 0.147 \\
\hline Current cannabis use & $0.94(0.55,1.58)$ & 0.804 & $1.09(0.30,3.90)$ & 0.896 \\
\hline Ever used amphetamine & $1.36(0.75,2.47)$ & 0.303 & $1.96(0.57,6.76)$ & 0.285 \\
\hline \multicolumn{5}{|l|}{ Leisure time sedentary behaviour/day } \\
\hline$<3 \mathrm{~h}$ & 1 (Reference) & & 1 (Reference) & \\
\hline $3-4 h$ & $0.75(0.47,1.19)$ & 0.214 & $1.26(0.83,1.90)$ & 0.273 \\
\hline $5-7 \mathrm{~h}$ & $1.28(0.88,1.87)$ & 0.193 & $2.06(1.25,3.41)$ & 0.005 \\
\hline$\geq 8 \mathrm{~h}$ & $1.36(0.80,2.31)$ & 0.250 & $1.91(1.14,3.20)$ & 0.014 \\
\hline
\end{tabular}

Injury in past 12 months 
Table 4 Associations with psychological distress among males and females (Continued)

\begin{tabular}{|c|c|c|c|c|}
\hline \multirow[t]{2}{*}{ Variable } & \multicolumn{2}{|l|}{ Male } & \multicolumn{2}{|l|}{ Female } \\
\hline & Adjusted Odds Ratio (95\% Cl) & $p$-value & Adjusted Odds Ratio (95\% Cl) & $p$-value \\
\hline 0 times & 1 (Reference) & & 1 (Reference) & \\
\hline 1 time & $1.55(1.18,2.04)$ & 0.002 & $1.37(0.95,1.97)$ & 0.090 \\
\hline 2 or more times & $1.75(1.27,2.42)$ & $<0.001$ & $3.09(1.73,5.48)$ & $<0.001$ \\
\hline \multicolumn{5}{|l|}{ Soft drink intake/day } \\
\hline 0 & 1 (Reference) & & 1 (Reference) & \\
\hline 1 & $1.18(0.76,1.84)$ & 0.459 & $0.70(0.50,0.99)$ & 0.043 \\
\hline 2 & $1.56(1.00,2.45)$ & 0.050 & $0.63(0.33,1.20)$ & 0.160 \\
\hline 3 or more & $1.13(0.56,2.29)$ & 0.729 & $0.89(0.52,1.52)$ & 0.664 \\
\hline \multicolumn{5}{|c|}{ Fast food consumption/week } \\
\hline 0 days & 1 (Reference) & & 1 (Reference) & \\
\hline 1 & $1.08(0.72,1.65)$ & 0.689 & $0.80(0.52,1.22)$ & 0.293 \\
\hline 2 & $0.98(0.67,1.43)$ & 0.905 & $0.77(0.47,1.27)$ & 0.302 \\
\hline $3-7$ days & $0.89(0.62,1.27)$ & 0.515 & $1.13(0.75,1.71)$ & 0.549 \\
\hline \multicolumn{5}{|c|}{ Fruit and vegetable intake } \\
\hline$<1$ serving/day & 1 (Reference) & & 1 (Reference) & \\
\hline 1 or 2 & $1.11(0.65,1.91)$ & 0.705 & $0.99(0.62,1.59)$ & 0.573 \\
\hline 3 or 4 & $0.95(0.58,1.57)$ & 0.852 & $0.86(0.56,1.32)$ & 0.494 \\
\hline 5 or more & $1.16(0.67,2.00)$ & 0.595 & $0.79(0.51,1.23)$ & 0.294 \\
\hline
\end{tabular}

Cl Confidence Interval

sedentary behaviour, for example social media use, we are not able to show the potentially negative effects of social media use on psychological distress [46]. In a systematic review among adolescents [47] found that sedentary behaviour was associated with poor mental health and psychological distress, which may be explained by "the beneficial pathophysiological, social and general health effects of being active may be omitted when sedentary, which may have a negative impact on mental health." Another possible mechanism by which sedentary behaviour may increase psychological distress is via inflammatory processes [48]. For example, in a randomized controlled intervention, "a one-week sedentary behaviour-inducing intervention had deleterious effects on anxiety in an active, young adult population" [49]. The association between injury occurrence in the past 12 months and psychological distress in the past 12 months may be explained by "the injury occurrence being considered a particularly impactful stressful life event, and experiences of stressful life events have been strongly associated with prospective anxiety symptom development" [50].

\section{Study limitations}

Study limitations include that this investigation was limited because of its cross-sectional design, the inclusion of only school adolescents as well as the self-report of the data. An additional limitation was that the GSHS in Morocco only assessed psychological distress with two items which may not reflect a standardized scale nor a diagnostic interview, and did not assess help seeking behaviours for psychological distress.

\section{Conclusion}

The study found among school-going nationally representative adolescents in Morocco that almost one in four students reported psychological distress. Several correlates for psychological distress were identified, including female sex, having no close friends, older age, bullying victimization, infrequently physically attacked, frequent participation in physical fights, frequent experience of hunger, parental emotional neglect, parental disrespect of privacy, school truancy, sedentary behaviour and having sustained a single or multiple serious injuries (past year), which can potentially guide interventions to prevent psychological distress in this adolescent school population.

\section{Supplementary information}

Supplementary information accompanies this paper at https://doi.org/10 1186/s12888-020-02888-3.

Additional file 1. Variable description. 


\section{Abbreviations}

GSHS: Global School-Based Student Health Survey; STATA: Statistics and data

\section{Acknowledgements}

The data source, the World Health Organization NCD Microdata Repository (URL: https://extranet.who.int/ncdsmicrodata/index.php/catalog), is hereby acknowledged.

\section{Authors' contributions}

All authors fulfil the criteria for authorship. SP and KP conceived and designed the research, performed statistical analysis, drafted the manuscript and made critical revision of the manuscript for key intellectual content. All authors read and approved the final version of the manuscript and have agreed to authorship and order of authorship for this manuscript.

\section{Funding}

Not applicable.

\section{Availability of data and materials}

The data for the current study are publicly available at the World Health Organization NCD Microdata Repository (URL: https://extranet.who.int/ ncdsmicrodata/index.php/catalog).

\section{Ethics approval and consent to participate}

The present study was based on an analysis of the Morocco 2016 GSHS survey dataset freely available online with all identifier information detached. The Morocco 2016 GSHS was approved by the School and University Health Division, Ministry of Health, Rabat, Morocco and the World Health Organization. Therefore, the permission and ethical approval for the present analysis was automatically deemed unnecessary. Moreover, during the GSHS survey, written assent attached to a questionnaire was obtained from all eligible participants before filling the questionnaire.

\section{Consent for publication}

Not applicable.

\section{Competing interests}

The authors declare that they have no competing interests.

\section{Author details}

${ }^{1}$ ASEAN Institute for Health Development, Mahidol University, Salaya, Phutthamonthon, Nakhon Pathom, Thailand. ${ }^{2}$ Department of Research Administration and Development, University of Limpopo, Polokwane, South Africa. ${ }^{3}$ Department of Psychology, University of the Free State, Bloemfontein, South Africa.

Received: 7 May 2020 Accepted: 21 September 2020

\section{Published online: 29 September 2020}

\section{References}

1. World Health Organization (WHO). Adolescent mental health; 2019. URL: https://www.who.int/news-room/fact-sheets/detail/adolescent-mentalhealth (Accessed 2 Jan 2020).

2. Polanczyk GV, Salum GA, Sugaya LS, Caye A, Rohde LA. Annual research review: a meta-analysis of the worldwide prevalence of mental disorders in children and adolescents. J Child Psychol Psychiatry. 2015;56(3):345-65. https://doi.org/10.1111/jcpp.12381.

3. Kessler RC, Amminger GP, Aguilar-Gaxiola S, Alonso J, Lee S, Ustün TB. Age of onset of mental disorders: a review of recent literature. Curr Opin Psychiatry. 2007;20(4):359-64. https://doi.org/10.1097/YCO. Ob013e32816ebc8c.

4. Marsh IC, Chan SWY, MacBeth A. Self-compassion and psychological distress in adolescents-a meta-analysis. Mindfulness (N Y). 2018;9(4):1011-27. https:// doi.org/10.1007/s12671-017-0850-7

5. American Psychological Association (APA) Dictionary of Psychology: Psychological distress, 2020. URL https://dictionary.apa.org/psychologicaldistress.

6. Kessler RC, Barker PR, Colpe LJ, Epstein JF, Gfroerer JC, Hiripi E, et al. Screening for serious mental illness in the general population. Arch Gen Psychiatry. 2003;60(2):184-9. https://doi.org/10.1001/archpsyc.60.2.184.
7. Furukawa TA, Kessler RC, Slade T, Andrews G. The performance of the K6 and K10 screening scales for psychological distress in the Australian National Survey of mental health and well-being. Psychol Med. 2003;33(2): 357-62. https://doi.org/10.1017/s0033291702006700.

8. Goldberg DP, Blackwell B. Psychiatric illness in general practice. A detailed study using a new method of case identification. Br Med J. 1970;1:439-43.

9. Siziya S, Mazaba ML. Prevalence and correlates for psychosocial distress among in-school adolescents in Zambia. Front Public Health. 2015;3:180. https://doi.org/10.3389/fpubh.2015.00180.

10. Pengpid S, Peltzer K. Psychological distress and its associated factors among school-going adolescents in Tanzania. Psychol Stud. 2020;65(2):174. https:// doi.org/10.1007/s12646-020-00550-2.

11. Pengpid S, Peltzer K. High psychological distress among school-going adolescents in Afghanistan: prevalence and correlates from a national survey. Vulnerable Child Youth Stud. 2020;15(1):40-7. https://doi.org/10. 1080/17450128.2019.1679937.

12. Ismail A, Abdelgaber A, Hegazi H, Lotfi M, Kamel A, Ramdan M. The prevalence and risk factors of anxiety disorders in an Egyptian sample of school and students at the age of 12-18 years. J Psychiatry. 2015;18:316 10. 4172/2378- 5756.1000316.

13. Ahadi Z, Oorbani M, Kelishadi R, Ardalan G, Taslimi M, Mahmoudarabi M, et al. Regional disparities in psychiatric distress, violent behavior, and life satisfaction in Iranian adolescents: the CASPIAN-III study. J Dev Behav Pediatr. 2014;35(9):582-90. https://doi.org/10.1097/DBP.0000000000000103.

14. Khalid A, Qadir F, Chan SWY, Schwannauer M. Adolescents' mental health and well-being in developing countries: a cross-sectional survey from Pakistan. J Ment Health. 2019;28(4):389-96. https://doi.org/10.1080/ 09638237.2018 .1521919

15. Mwakanyamale AA, Yizhen Y. Psychological maltreatment and its relationship with self-esteem and psychological stress among adolescents in Tanzania: a community based, cross-sectional study. BMC Psychiatry. 2019;19(1):176. https://doi.org/10.1186/s12888-019-2139-y.

16. Jaisoorya TS, Geetha D, Beena KV, Beena M, Ellangovan K, Thennarasu K. Prevalence and correlates of psychological distress in adolescent students from India. East Asian Arch Psychiatr. 2017;27(2):56-62.

17. Arbour-Nicitopoulos KP, Faulkner GE, Irving HM. Multiple health-risk behaviour and psychological distress in adolescence. J Can Acad Child Adolesc Psychiatry. 2012;21(3):171-8.

18. Okwaraji FE, Obiechina Kl, Onyebueke GC, Udegbunam ON, Nnadum GS. Loneliness, life satisfaction and psychological distress among out-of-school adolescents in a Nigerian urban city. Psychol Health Med. 2018;23(9):110612. https://doi.org/10.1080/13548506.2018.147672.

19. Lee H, Lee EY, Greene B, Shin YJ. Psychological distress among adolescents in Laos, Mongolia, Nepal, and Sri Lanka. Asian Nurs Res (Korean Soc Nurs Sci). 2019;13(2):147-53. https://doi.org/10.1016/j.anr.2019.04.001.

20. Zouini B, Sfendla A, Hedman Ahlström B, Senhaji M, Kerekes N. Mental health profile and its relation with parental alcohol use problems and/or the experience of abuse in a sample of Moroccan high school students: an explorative study. Ann General Psychiatry. 2019;18:27. https://doi.org/10. 1186/s12991-019-0251-5

21. Kieling $\mathrm{C}$, Baker-Henningham $\mathrm{H}$, Belfer $\mathrm{M}$, et al. Child and adolescent mental health worldwide: evidence for action. Lancet. 2011;378(9801):1515-25. https://doi.org/10.1016/S0140-6736(11)60827-1.

22. Khan MMA, Rahman MM, Islam MR, Karim M, Hasan M, Jesmin SS. Suicidal behavior among school-going adolescents in Bangladesh: findings of the global school-based student health survey. Soc Psychiatry Psychiatr Epidemiol. 2020. https://doi.org/10.1007/s00127-020-01867-z.

23. World Health Organization (WHO). Global school-based student health survey (GSHS); 2019. URL: https://www.who.int/ncds/surveillance/gshs/en/ (Accessed 10 April 2020).

24. Kroenke K, Spitzer RL, Williams JB. The patient health Questionnaire-2: validity of a two-item depression screener. Med Care. 2003:41(11):1284-92. https://doi.org/10.1097/01.MLR.0000093487.78664.3C

25. Kroenke K, Spitzer RL, Williams JB, Monahan PO, Löwe B. Anxiety disorders in primary care: prevalence, impairment, comorbidity, and detection. Ann Intern Med. 2007;146(5):317-25. https://doi.org/10.7326/0003-4819-146-5200703060-00004

26. Plummer F, Manea L, Trepel D, McMillan D. Screening for anxiety disorders with the GAD-7 and GAD-2: a systematic review and diagnostic metaanalysis. Gen Hosp Psychiatry. 2016:39:24-31. https://doi.org/10.1016/j. genhosppsych.2015.11.005. 
27. World Health Organization (WHO). Adverse childhood experiences international questionnaire (ACE-IQ) guidance for Analysing ACE-IQ; 2016. URL: https://www.who.int/violence_injury_prevention/violence/activities/ adverse_childhood_experiences/guidance_for_analysing.pdf?ua=1 (Accessed 2 April 2020).

28. Al-Yateem N, Bani Issa W, Rossiter RC, Al-Shujairi A, Radwan H, Awad M, et al. Anxiety related disorders in adolescents in the United Arab Emirates: a population based cross-sectional study. BMC Pediatr. 2020;20(1):245. https:// doi.org/10.1186/s12887-020-02155-0.

29. Maalouf FT, Ghandour LA, Halabi F, Zeinoun P, Shehab AA, Tavitian L. Psychiatric disorders among adolescents from Lebanon: prevalence, correlates, and treatment gap. Soc Psychiatry Psychiatr Epidemiol. 2016; 51(8):1105-16. https://doi.org/10.1007/s00127-016-1241-4.

30. Angold A, Erkanli A, Silberg J, Eaves L, Costello EJ. Depression scale scores in 8-17-year-olds: effects of age and gender. J Child Psychol Psychiatry. 2002; 43(8):1052-63. https://doi.org/10.1111/1469-7610.00232.

31. Rescorla L, Achenbach TM, Ivanova MY, Dumenci L, Almqvist F, Bilenberg N, et al. Epidemiological comparisons of problems and positive qualities reported by adolescents in 24 countries. J Consult Clin Psychol. 2007;75(2): 351-8. https://doi.org/10.1037/0022-006X.75.2.351.

32. Byrne DG, Davenport SC, Mazanov J. Profiles of adolescent stress: the development of the adolescent stress questionnaire (ASQ). J Adolesc. 2007; 30(3):393-416. https://doi.org/10.1016/j.adolescence.2006.04.004.

33. Spear LP. The adolescent brain and age-related behavioral manifestations. Neurosci Biobehav Rev. 2000;24(4):417-63. https://doi.org/10.1016/s01497634(00)00014-2.

34. Landstedt E, Gillander GK. Experiences of violence among adolescents: gender patterns in types, perpetrators and associated psychological distress. Int J Public Health. 2011;56(4):419-27. https://doi.org/10.1007/s00038-0110258-4.

35. Fleming LC, Jacobsen KH. Bullying among middle-school students in low and middle income countries. Health Promot Int. 2010;25(1):73-84. https:// doi.org/10.1093/heapro/dap046.

36. Kazarian SS, Ammar J. School bullying in the Arab world: a review. Arab J Psychiatry. 2013;24(1):37-45. https://doi.org/10.12816/0000097.

37. Save the Children (Middle East and Eastern Europe Regional Office) and UNICEF (MENA Regional Office) November 2017 Violence against adolescents and youth: new evidence and key policy issues for Mena. URL: https://www.menayouthhub.org/sites/menayouthhub.org/files/201903/51\%20Violence\%20Evidence\%20Paper\%20ESAY\%202017.pdf.

38. Beattie TS, Prakash R, Mazzuca A, Kelly L, Javalkar P, Raghavendra T, et al. Prevalence and correlates of psychological distress among 13-14 year old adolescent girls in North Karnataka, South India: a cross-sectional study. BMC Public Health. 2019;19(1):48. https://doi.org/10.1186/s12889-018-6355-z.

39. Tabak I, Jodkowska M, Oblacińska A. Społeczne uwarunkowania podwyzszonego poziomu stresu psychologicznego u młodziezy 18-letniej w Polsce [social determinants of psychological distress in adolescents aged 18 years in Poland]. Med Wieku Rozwoj. 2008;12(2 Pt 1):569-76.

40. Klasen $\mathrm{H}, \mathrm{Crombag}$ AC. What works where? A systematic review of child and adolescent mental health interventions for low and middle income countries. Soc Psychiatry Psychiatr Epidemiol. 2013;48(4):595-611. https:// doi.org/10.1007/s00127-012-0566-X.

41. Hong SA, Peltzer K. Dietary behaviour, psychological well-being and mental distress among adolescents in Korea. Child Adolesc Psychiatry Ment Health. 2017;11:56. https://doi.org/10.1186/s13034-017-0194-z.

42. Jacob L, Smith L, Haro JM, Stickley A, Koyanagi A. Serious physical injury and depressive symptoms among adolescents aged 12-15 years from 21 low- and middle-income countries. J Affect Disord. 2020;264:172-80. https:// doi.org/10.1016/j.jad.2019.12.026.

43. Pengpid S, Peltzer K. Early substance use initiation and psychological distress among adolescents in five ASEAN countries: a cross-sectional study. Psychol Res Behav Manag. 2019;12:1003-8. https://doi.org/10.2147/PRBM. S223624.

44. Pengpid S, Peltzer K. Leisure-time sedentary behavior is associated with psychological distress and substance use among school-going adolescents in five southeast Asian countries: a cross-sectional study. Int J Environ Res Public Health. 2019;16(12):2091. https://doi.org/10.3390/ijerph16122091.

45. Williams JL, Rheingold AA, Knowlton AW, Saunders BE, Kilpatrick DG, Associations between motor vehicle crashes and mental health problems: data from the National Survey of adolescents-replication. J Trauma Stress. 2015;28(1):41-8. https://doi.org/10.1002/jts.21983.
46. Keles B, McCrae N. \& Grealish. A systematic review: the influence of social media on depression, anxiety and psychological distress in adolescents. Int J Adolesc Youth. 2020;25(1):79-93. https://doi.org/10.1080/02673843.2019. 1590851.

47. Hoare E, Milton K, Foster C, Allender S. The associations between sedentary behaviour and mental health among adolescents: a systematic review. Int J Behav Nutr Phys Act. 2016;13(1):108. https://doi.org/10.1186/s12966-0160432-4.

48. Vancampfort D, Van Damme T, Stubbs B, Smith L, Firth J, Hallgren M, et al. Sedentary behavior and anxiety-induced sleep disturbance among 181,093 adolescents from 67 countries: a global perspective. Sleep Med. 2019;58:1926. https://doi.org/10.1016/j.sleep.2019.01.048.

49. Edwards MK, Loprinzi PD. Experimentally increasing sedentary behavior results in increased anxiety in an active young adult population. J Affect Disord. 2016;204:166-73. https://doi.org/10.1016/j.jad.2016.06.045.

50. McLaughlin KA, Hatzenbuehler ML. Stressful life events, anxiety sensitivity, and internalizing symptoms in adolescents. J Abnorm Psychol. 2009;118(3): 659-69. https://doi.org/10.1037/a0016499.

\section{Publisher's Note}

Springer Nature remains neutral with regard to jurisdictional claims in published maps and institutional affiliations.
Ready to submit your research? Choose BMC and benefit from:

- fast, convenient online submission

- thorough peer review by experienced researchers in your field

- rapid publication on acceptance

- support for research data, including large and complex data types

- gold Open Access which fosters wider collaboration and increased citations

- maximum visibility for your research: over $100 \mathrm{M}$ website views per year

At BMC, research is always in progress.

Learn more biomedcentral.com/submissions 